\title{
DEXTRANAS EM AÇÚCARES DO ESTADO DE SÃO PAULO
}

\author{
Francisco Wendel Batista de Aquino e Douglas Wagner Franco* \\ Instituto de Química de São Carlos, Universidade de São Paulo, CP 780, 13560-970 São Carlos - SP, Brasil
}

Recebido em 15/3/07; aceito em 27/11/07; publicado na web em 8/7/08

\begin{abstract}
DEXTRANS IN SUGARS FROM SÃO PAULO STATE. The characterization of dextran in sugars by size exclusion chromatography (SEC) has been carried out according to the number-average molecular weight (Mn), the weight-average molecular weight (Mw), the Z-average molecular weight $(\mathrm{Mz})$, and the polydispersity $(\mathrm{Mw} / \mathrm{Mn})$. The results suggest that all the analyzed thirty sugar samples from São Paulo state were contaminated with two or three different dextran polymers. The collected data clearly point out that the total dextran content together with the mass distribution parameters $\mathrm{Mw}, \mathrm{Mn}, \mathrm{Mz}$, and $\mathrm{Mw} / \mathrm{Mn}$ should be considered during the evaluation of the quality of the sugar used for the cachaça sweeting process.
\end{abstract}

Keywords: dextrans; characterization; cachaça.

\section{INTRODUÇÃO}

As dextranas constituem uma classe de polímeros de fórmula empírica $\left(\mathrm{C}_{6} \mathrm{H}_{10} \mathrm{O}_{5}\right)_{\mathrm{n}}$ cujo monômero é o $\alpha$-D-glucaconopiranosil. ${ }^{1,2}$ São contaminantes endêmicos da sacarose, originados principalmente devido à presença de bactérias do gênero Leuconostoc comumente presentes nas plantações de cana-de-açúcar. ${ }^{2-4}$

A massa molar média (Mw) das dextranas pode variar de 1.500 até vários milhões de daltons $(\mathrm{Da})$, e a sua estrutura dependerá principalmente da espécie bacteriana e do tipo de dextranasacarase presente no meio. ${ }^{5}$ Normalmente as dextranas produzidas pelas cepas mais comuns do Leuconostoc mesenteroides apresentam mais de $90 \%$ de ligações glicosídicas $\alpha(1 \rightarrow 6)$, sendo o restante de suas ligações do tipo $\alpha(1 \rightarrow 4), \alpha(1 \rightarrow 3), \alpha(1 \rightarrow 2)$, as quais são responsáveis pelas ramificações ao longo da cadeia. ${ }^{1,2,6}$

As ramificações, além de influenciarem diretamente na extensão da cadeia polimérica, atuam conjuntamente com a variação da massa molar média nas propriedades físico-químicas das dextranas, como viscosidade intrínseca, solubilidade em água e em soluções hidroalcoólicas e, conseqüentemente, nas características do seu precipitado. ${ }^{1,5,7}$

Embora inócuas à saúde humana e com aplicações variadas, ${ }^{1}$ a presença das dextranas em açúcares está associada a problemas que conduzem a perdas na produção. ${ }^{4,5,8}$ Sua presença em açúcares utilizados na adoçagem de bebidas alcoólicas é responsável pela formação de precipitados ${ }^{4,8}$ denominados flocos alcoólicos, os quais são considerados defeitos sensoriais. ${ }^{9}$

Estes precipitados podem ainda ser consequiência de alterações estruturais nas dextranas por associação a íons metálicos. ${ }^{10,11}$

É bem estabelecida a precipitação de dextranas em função de sua massa molar e do percentual de etanol presente em diferentes matrizes. ${ }^{4,12}$ A relação massa molar versus concentração de etanol é o fundamento de várias metodologias para a análise quantitativa de dextranas, tais como os métodos de Robert's ${ }^{4,9}$ e Haze. ${ }^{4}$

Apesar da concentração de sacarose adicionada na cachaça ser regulamentada, ${ }^{13}$ a sua concentração não é informada no rótulo do produto, e pouco se sabe a respeito das características estruturais das dextranas presentes no açúcar utilizado pelos produtores de cachaça.

No intuito de contribuir para o melhor conhecimento da quali-

*e-mail: douglas@iqsc.usp.br dade do açúcar utilizado na adoçagem da cachaça, este trabalho descreve o perfil das dextranas presentes em amostras de sacarose em função da sua massa molar média ponderal (Mw), massa molar numérica média $(\mathrm{Mn})$, massa molar média $(\mathrm{Mz})$ e índice de polidispersidade $(\mathrm{Mw} / \mathrm{Mn})$.

\section{PARTE EXPERIMENTAL}

\section{Instrumentação e reagentes}

As análises cromatográficas por exclusão por tamanho (SEC), foram realizadas em um cromatógrafo líquido Shimadzu, constituído por um módulo controlador SLC-10AVP, duas bombas de alta pressão LC-10AD, um detector de índice de refração Shimadzu RID-10A e um loop Rheodyne de $100 \mu \mathrm{L}$. O sistema foi operado em modo isocrático com fluxo de $0,8 \mathrm{~mL} / \mathrm{min}$ de $\mathrm{Na}_{2} \mathrm{SO}_{4} 0,5 \mathrm{M}$. As separações foram efetuadas em três colunas Waters Ultrahydrogel linear $(7,8 \times 300 \mathrm{~mm})$ conectadas em série.

$\mathrm{Na}$ etapa de isolamento das dextranas foi utilizada uma ultracentrífuga Hitachi Himac CR20b2.

A aquisição e o tratamento dos dados cromatográficos foram realizados utilizando, respectivamente, os softwares Shimadzu Class-VP 6.12 e GPC for Class-Vp 1.02.

Os padrões de dextranas foram adquiridos da American Polymer (EUA), o etanol anidro e o sulfato de sódio da Mallinckrodt (EUA). Das 30 amostras analisadas, 25 foram cedidas pelo Centro de Tecnologia Canavieira - CTC (Piracicaba-SP) o qual certificou que estas apresentam o mesmo padrão de qualidade das adquiridas pelos produtores de cachaça, e 5 foram obtidas junto a pequenos produtores.

\section{Metodologia}

O preparo das amostras seguiu o seguinte procedimento: em um balão volumétrico de 50,0 mL, 4,00 g do açúcar foram dissolvidas em 10,0 mL de água tipo Milli Q. Em seguida o volume foi completado com etanol anidro, resultando uma solução hidroalcoólica $80 \%$ de etanol v/v. Este procedimento foi adotado visando garantir a precipitação das dextranas de alta e baixa massa molar. ${ }^{4}$

Todo o volume do balão foi transferido em partes iguais para dois tubos de centrífuga de $50 \mathrm{~mL}$ com tampa. Decorrido um período de repouso de $24 \mathrm{~h}$ estes foram centrifugados a $15.000 \mathrm{rpm}$ a 
temperatura de $4{ }^{\circ} \mathrm{C}$ por $2 \mathrm{~h}$.

Após a centrifugação dispensou-se a fase líquida e ao precipitado de cada tubo foi adicionado $1,0 \mathrm{~mL}$ de $\mathrm{Na}_{2} \mathrm{SO}_{4} 0,5 \mathrm{M}$ para a solubilização das dextranas. As soluções resultantes foram transferidas para um único frasco que foi mantido sob refrigeração por $24 \mathrm{~h}$. Decorrido este período a solução foi filtrada em membrana de éster de celulose $(\varnothing 0,45 \mu \mathrm{m})$, e uma alíquota de $100,0 \mu \mathrm{L}$ do filtrado foi injetada no sistema cromatográfico.

Para a caracterização das dextranas foram empregados padrões com as seguintes massas molares médias: $5,2 \times 10^{3}, 2,38 \times 10^{4}$, $1,48 \times 10^{5}, 2,73 \times 10^{5}, 4,10 \times 10^{5}, 1,10 \times 10^{6}, 2,10 \times 10^{6}, 4,20 \times 10^{6}$, $5,90 \times 10^{6}$ e $7,40 \times 10^{6}$ Da.

Para avaliar o melhor tipo de curva de calibração, os tempos de retenção $\left(\mathrm{t}_{\mathrm{r}}\right)$ dos padrões foram substituídos em diferentes equações de calibração disponibilizadas pelo software GPC for Classvp. A calibração com a equação $\log \mathrm{Mw}=a x^{3}+b x^{2}+c x+d$, com $x=t$, foi escolhida por apresentar o menor valor de dispersão dos padrões em relação à curva ideal $(\mathrm{d}=0,0927)$.

A curva de calibração ilustrada na Figura 1 (Mw: 5,2 x $10^{3}$ até $7,4 \times 10^{6} \mathrm{Da}$ ), foi construída utilizando colunas contendo na fase estacionária uma mistura de partículas com tamanho de poro de 120 até $2000 \AA$ A, possibilitando uma ampla faixa de trabalho.



Figura 1. Curva de calibração utilizada na caracterização das dextranas, presentes nas amostras de açúcar analisadas

\section{RESULTADOS E DISCUSSÃO}

A presença de amido, eventualmente precipitado, em conjunto com as dextranas pela adição de etanol foi considerada. É conhecida a baixa solubilidade do amido em água fria ${ }^{14-16}$ e do mesmo se apresentar em grânulos com diâmetro de 1-100 $\mu \mathrm{m} .{ }^{14,16}$ Assim, os precipitados obtidos pela adição de etanol em cada amostra, após sua separação, foram redissolvidos em $\mathrm{Na}_{2} \mathrm{SO} 40,5 \mathrm{M}_{\text {(aq) }}$ e as soluções resultantes foram filtradas utilizando-se membranas com diâmetro de $0,45 \mu \mathrm{m}$.

Solução de iodo $0,5 \%$ foi então adicionada às membranas e a alíquotas do filtrado. Apenas nas membranas observou-se o desenvolvimento da coloração azul típica do complexo amido-iodo, ${ }^{17}$ descartando a presença de amido nas soluções submetidas à análise cromatográfica.

A Figura 2 apresenta um cromatograma típico das amostras de açúcar analisadas. Conforme observado na Figura 2, as amostras de açúcar apresentam dextranas com diferentes valores de massa molar, sendo que em todas as amostras foram observadas pelo menos duas dextranas distintas, de acordo com os dados apresentados na Tabela 1.



Figura 2. Cromatograma típico de dextranas presentes numa amostra de

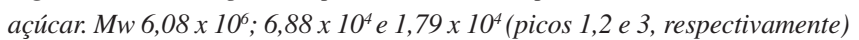

Os valores de massa molar média ponderal (Mw), massa molar numérica $(\mathrm{Mn})$, massa molar média $(\mathrm{Mz})$ e índice de polidispersidade são reconhecidos parâmetros para avaliar características de tamanho e linearidade de polímeros, ${ }^{18}$ tais como as dextranas presentes em açúcares. ${ }^{4,5,19}$

Dados sobre a polidispersividade (razão $\mathrm{Mw} / \mathrm{Mn}$ ) propiciam avaliar a amplitude da distribuição das massas molares de um polimero. ${ }^{18}$ Dextranas mais polidispersas apresentam uma maior amplitude na sua distribuição de massa, tendendo assim a apresentar um maior número de ramificações, sendo bem estabelecido que a solubilidade das dextranas em soluções hidroalcoólicas e dependendo da bactéria produtora, mesmo em água, diminui à medida que aumenta o número de ramificações. . $^{518-20}$

As massas molares de polímeros devem ser expressas em termos médios, uma vez que suas curvas de distribuição de massas molares (DPM) são relativamente largas. A massa molar média (Mz) é mais sensível às frações de mais alta massa de um polímero, que a massa molar média ponderal $(\mathrm{Mw})$ e a massa numérica $(\mathrm{Mn}){ }^{18}$ Isto torna importante o seu conhecimento em usinas, pois dextranas com alta Mz estão associadas a problemas como entupimento de filtros, interferências na remoção de partículas suspensas durante a etapa de clarificação do açúcar bruto e aumento da viscosidade do caldo, o que reduz a eficiência de cristalização da sacarose. ${ }^{5}$

Com relação à cachaça, dextranas mais polidispersas e com massa molar mais elevada tenderão a precipitar mais rapidamente mesmo em destilados de menor teor alcoólico.

Conforme a Tabela 1, dextranas com alta massa molar média ponderal (média $=5.100 .080 \mathrm{Da}$ ) estão presentes em todas as amostras. O que é esperado, pois dextranas com massa molar da ordem de 5.000.000 Da e polidispersas (valores de $\mathrm{Mw}>\mathrm{Mn}$ ), ${ }^{21}$ são consideradas evidências da presença das espécies mais comuns do Leuconostoc em plantações de cana. ${ }^{21}$

Ainda com relação à Tabela 1, em 96,7\% das amostras foi identificado um segundo grupo de dextranas com massas molares inferiores às do primeiro grupo (média $41.052 \mathrm{Da}$ ). Um terceiro grupo (média $13.389 \mathrm{Da}$ ) está presente em $36,7 \%$ das amostras.

Não se pode atribuir a presença destas dextranas de menor massa molar a uma única causa. Kim e colaboradores ${ }^{6}$ demonstraram que uma mesma cepa do Leuconostoc pode produzir simultaneamente dextranas com diferentes massas molares, cujas proporções serão função da concentração de sacarose, do $\mathrm{pH}$ e da temperatura do meio. Em altas concentrações de sacarose, as dextranas produzidas pelo Leuconostoc apresentam uma distribuição bimodal entre baixa e alta massa molar $\left(10^{3}-10^{4}\right.$ e $\left.10^{6} \mathrm{Da}\right)$, respectivamente. ${ }^{6}$ 
Tabela 1. Perfil da distribuição de massas molares das dextranas nas amostras de açúcar analisadas

\begin{tabular}{|c|c|c|c|c|c|c|c|c|c|c|c|c|}
\hline \multirow[b]{2}{*}{ Amostra } & \multicolumn{4}{|c|}{ Dextrana 1} & \multicolumn{4}{|c|}{ Dextrana 2} & \multicolumn{4}{|c|}{ Dextrana 3} \\
\hline & $\begin{array}{c}\text { Massa } \\
\text { molar } \\
\text { numérica } \\
(\mathrm{Mn})\end{array}$ & $\begin{array}{l}\text { Massa } \\
\text { molar } \\
\text { média } \\
(\mathrm{Mw})\end{array}$ & $\begin{array}{cc}\text { Massa } & P \\
\text { molar } & \\
(\mathrm{Mz}) & \end{array}$ & $\begin{array}{l}\text { Polidisper- } \\
\text { sividade } \\
(\mathrm{Mw} / \mathrm{Mn})\end{array}$ & $\begin{array}{c}\text { Massa } \\
\text { molar } \\
\text { numérica } \\
(\mathrm{Mn})\end{array}$ & $\begin{array}{l}\text { Massa } \\
\text { molar } \\
\text { média } \\
(\mathrm{Mw})\end{array}$ & $\begin{array}{c}\text { Massa } \\
\text { molar } \\
(\mathrm{Mz})\end{array}$ & $\begin{array}{l}\text { Polidisper- } \\
\text { sividade } \\
(\mathrm{Mw} / \mathrm{Mn})\end{array}$ & $\begin{array}{c}\text { Massa } \\
\text { molar } \\
\text { numérica } \\
(\mathrm{Mn})\end{array}$ & $\begin{array}{c}\text { Massa } \\
\text { molar } \\
\text { média } \\
(\mathrm{Mw})\end{array}$ & $\begin{array}{c}\text { Massa } \\
\text { molar } \\
(\mathrm{Mz})\end{array}$ & $\begin{array}{c}\text { Polidisper- } \\
\text { sividade } \\
\text { (Mw/Mn) }\end{array}$ \\
\hline 1 & 1.118 .520 & 3.245 .905 & 6.681 .120 & 2,90 & 15.557 & 24.418 & 39.170 & 1,57 & & & & \\
\hline 2 & 4.442 .255 & 6.199 .935 & 8.551 .340 & 1,40 & 52.030 & 58.001 & 64.697 & 1,11 & & & & \\
\hline 3 & 1.940 .870 & 4.397 .855 & 6.784 .230 & 2,27 & 12.193 & 13.727 & 15.584 & 1,13 & & & & \\
\hline 4 & 4.599 .180 & 5.618 .515 & 6.795 .140 & 1,22 & 20.401 & 32.424 & 53.658 & 1,59 & & & & \\
\hline 5 & 4.537 .125 & 5.597 .235 & 6.812 .415 & 1,23 & 19.945 & 30.243 & 46.086 & 1,52 & & & & \\
\hline 6 & 4.127 .660 & 5.946 .590 & 8.501 .295 & 1,44 & 7.273 & 24.836 & 42.468 & 3,41 & & & & \\
\hline 7 & 1.472 .720 & 3.627 .260 & 6.361 .355 & 2,46 & 56.284 & 61.839 & 68.073 & 1,10 & 13.313 & 14.094 & 14.978 & 1,0586645 \\
\hline 8 & 5.242 .275 & 6.025 .300 & 6.907 .505 & 1,15 & 52.933 & 58.493 & 64.594 & 1,11 & 12.801 & 13.250 & 13.698 & 1,0350754 \\
\hline 9 & 2.517 .990 & 4.731 .240 & 6.923 .050 & 1,88 & 19.540 & 32.825 & 52.887 & 1,68 & & & & \\
\hline 10 & 5.223 .190 & 6.082 .520 & 7.054 .205 & 1,16 & 50.687 & 59.238 & 70.462 & 1,17 & 12.121 & 13.888 & 13.987 & 1,1457801 \\
\hline 11 & 5.567 .550 & 6.083 .355 & 6.620 .545 & 1,09 & 41.315 & 68.808 & 83.731 & 1,67 & 12.107 & 17.910 & 64.141 & 1,4793095 \\
\hline 12 & 3.397 .310 & 6.084 .045 & 10.027 .140 & 1,79 & 13.111 & 13.673 & 14.272 & 1,04 & & & & \\
\hline 13 & 2.494 .730 & 4.236 .410 & 5.887 .480 & 1,70 & 13.287 & 14.122 & 15.068 & 1,06 & & & & \\
\hline 14 & 4.937 .251 & 6.072 .835 & 7.442 .820 & 1,23 & 52.029 & 58.554 & 65.836 & 1,13 & 11.722 & 12.204 & 12.750 & 1,0411193 \\
\hline 15 & 1.163 .370 & 3.233 .595 & 6.117 .410 & 2,78 & 11.751 & 19.373 & 27.518 & 1,65 & & & & \\
\hline 16 & 2.668 .075 & 3.908 .180 & 5.373 .405 & 1,46 & 53.455 & 61.309 & 70.639 & 1,15 & 11.340 & 11.758 & 12.222 & 1,0368607 \\
\hline 17 & 4.866 .940 & 5.759 .240 & 6.767 .905 & 1,18 & 29.486 & 38.251 & 40.599 & 1,30 & & & & \\
\hline 18 & 4.751 .890 & 6.081 .630 & 7.712 .635 & 1,28 & 30.494 & 61.207 & 65.589 & 2,01 & 11.203 & 12.160 & 13.246 & 1,0854235 \\
\hline 19 & 2.041 .805 & 5.222 .485 & 11.892 .600 & 2,56 & 12.484 & 21.263 & 197.824 & 1,70 & & & & \\
\hline 20 & 4.665 .845 & 5.748 .525 & 7.030 .370 & 1,23 & & & & & & & & \\
\hline 21 & 1.582 .145 & 3.904 .165 & 6.751 .915 & 2,47 & 55.965 & 60.886 & 66.194 & 1,09 & 13.463 & 14.145 & 14.909 & 1,0506574 \\
\hline 22 & 1.427 .940 & 3.703 .230 & 6.650 .935 & 2,59 & 16.182 & 24.446 & 38.198 & 1,51 & & & & \\
\hline 23 & 2.887 .040 & 4.241 .315 & 5.956 .185 & 1,47 & 59.309 & 65.776 & 73.284 & 1,11 & 11.998 & 12.637 & 13.343 & 1,0532589 \\
\hline 24 & 3.488 .945 & 5.099 .945 & 7.056.280 & 1,46 & 37.719 & 48.580 & 60.096 & 1,29 & & & & \\
\hline 25 & 3.640 .220 & 5.013 .590 & 6.516 .980 & 1,38 & 56.532 & 61.936 & 68.013 & 1,10 & 11.778 & 12.450 & 13.202 & 1,0570555 \\
\hline 26 & 2.329 .560 & 4.915 .390 & 7.510 .790 & 2,11 & 57.388 & 63.180 & 69.443 & 1,10 & 11.505 & 12.933 & 14.463 & 1,1241199 \\
\hline 27 & 2.139 .395 & 4.568 .905 & 7.069 .800 & 2,14 & 57.784 & 62.443 & 67.519 & 1,08 & 12.183 & 13.238 & 14.421 & 1,0865961 \\
\hline 28 & 4.020 .295 & 5.650 .620 & 7.541 .650 & 1,41 & 13.795 & 23.234 & 40.005 & 1,68 & & & & \\
\hline 29 & 3.492 .330 & 5.714 .333 & 9.787 .690 & 1,64 & 6.902 & 11.622 & 20.008 & 1,68 & & & & \\
\hline 30 & 4.184 .225 & 6.288 .240 & 34.426 .430 & 1,50 & 11.386 & 15.787 & 26.294 & 1,39 & & & & \\
\hline Média & 3.365 .622 & 5.100 .080 & 8.183 .754 & 1,72 & 32.318 & 41.052 & 56.131 & 1,42 & 12.128 & 13.389 & 17.947 & 1,10 \\
\hline
\end{tabular}

Apesar do Leuconostoc mesenteroides ser o principal responsável pela presença de dextranas em açúcar de cana-de-açúcar, o surgimento de dextranas com diferentes massas molares pode também ser atribuído a presença de outras espécies bacterianas como Lactobacillus e Streptococcus, ${ }^{5,6}$ as quais podem contaminar a cana ainda no campo ou nas usinas, por exemplo por meio da água de lavagem da cana.

Outra possível explicação para a presença das dextranas de menor massa molar (Mw $\left.\leq 5,0 \times 10^{4} \mathrm{Da}\right)$ pode ser atribuída à adição de dextranases no caldo de cana. Estas enzimas quando adicionadas durante o processo de fabricação do açúcar nas usinas hidrolisam as dextranas originando dextranas de menor massa molar e oligossacarídeos. ${ }^{22}$

O decréscimo no índice de polidispersividade $1,72 \rightarrow 1,42 \rightarrow$ 1,10 observado é coerente pois, o número de ramificações nas dextranas tende a diminuir simultaneamente com a redução da sua massa molar (Mw). ${ }^{6,23,24}$

Mesmo levando-se em consideração o caráter majoritariamente qualitativo da cromatografia por exclusão por tamanho, com detecção por índice de refração, em nossos experimentos padrões de dextranas com massas molares significativamente distintas apresentaram em função da área do pico, respostas da mesma ordem de grandeza para as mesmas concentrações de dextranas, como observado na Figura 3.

Assim, atribuindo-se à metodologia empregada um caráter semiquantitativo, foi possível estimar os percentuais relativos de cada fração de dextrana com relação ao total de dextranas presentes em uma amostra, conforme apresentado na Tabela 2. De acordo com estes dados, as proporções entre as dextranas nas amostras, na sequência decrescente de massa molar, foram de 47,9 e $52,1 \%$ e

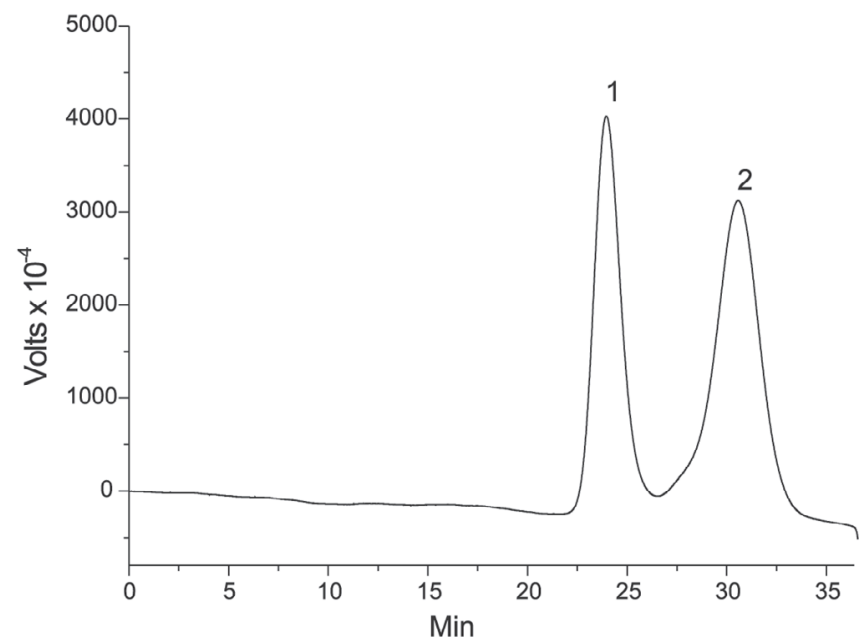

Figura 3. Cromatograma da mistura de 250 ppm dos padrões de dextranas Mw 5,90 × $10^{6}$ e $1,48 \times 10^{5}$ (picos 1 e 2 respectivamente) 
Tabela 2. Percentuais relativos para as dextranas determinadas nas 30 amostras de açúcar analisadas

\begin{tabular}{|c|c|c|c|c|}
\hline Amostra & $\%$ Dextrana 1 & $\%$ Dextrana 2 & $\%$ Dextrana 3 & C.V. \\
\hline 1 & 54,6 & 45,4 & & 8,8 \\
\hline 2 & 54,3 & 45,7 & & 6,9 \\
\hline 3 & 92,6 & 7,4 & & 10,6 \\
\hline 4 & 19,5 & 80,5 & & 7,7 \\
\hline 5 & 33,5 & 66,5 & & 6,3 \\
\hline 6 & 16,4 & 83,6 & & 11,2 \\
\hline 7 & 65,3 & 19,8 & 14,9 & 3,6 \\
\hline 8 & 7,7 & 46,3 & 46,0 & 4,8 \\
\hline 9 & 82,8 & 17,2 & & 8,8 \\
\hline 10 & 60,4 & 14,0 & 25,6 & 8,4 \\
\hline 11 & 25,3 & 31,6 & 43,1 & 5,7 \\
\hline 12 & 41,0 & 59,0 & & 5,8 \\
\hline 13 & 83,9 & 16,1 & & 5,5 \\
\hline 14 & 56,7 & 8,9 & 34,5 & 7,9 \\
\hline 15 & 15,5 & 84,5 & & 6,2 \\
\hline 16 & 35,0 & 41,5 & 23,6 & 7,4 \\
\hline 17 & 43,1 & 56,9 & & 11,3 \\
\hline 18 & 19,1 & 24,7 & 56,2 & 6,6 \\
\hline 19 & 37,3 & 62,7 & & 4,4 \\
\hline 20 & 100,0 & & & - \\
\hline 21 & 36,1 & 34,5 & 29,4 & 8,1 \\
\hline 22 & 62,1 & 37,9 & & 5,6 \\
\hline 23 & 27,8 & 42,8 & 29,4 & 4,5 \\
\hline 24 & 42,7 & 57,3 & & 3,5 \\
\hline 25 & 52,7 & 29,8 & 17,5 & 4,8 \\
\hline 26 & 83,6 & 4,8 & 11,6 & 8,4 \\
\hline 27 & 86,6 & 4,9 & 8,5 & 7,1 \\
\hline 28 & 54,6 & 45,4 & & 9,7 \\
\hline 29 & 46,6 & 53,4 & & 2,9 \\
\hline 30 & 34,4 & 65,6 & & 5,9 \\
\hline
\end{tabular}

46,$4 ; 25,3 ; 28,3 \%$, respectivamente.

Visando a eliminação de partículas sólidas no produto a ser envasado, a cachaça é geralmente filtrada à temperatura ambiente. Esta operação raramente é efetuada sob resfriamento e a maioria dos filtros empregados são mais apropriados para retenção de partículas macroscópicas, como é o caso dos filtros de areia. ${ }^{25}$

Filtros especiais como os à base de celulose, capazes de reter pequenas partículas, são utilizados pela minoria destas indústrias, em função do seu custo. De modo geral, é comum o emprego de filtros de eficiência bem inferior à de membranas com poros de $0,45 \mu \mathrm{m} .^{25}$

Procurando avaliar a eficiência da filtração no controle de dextranas, a soluções etanólicas $(40 \% \mathrm{v} / \mathrm{v})$ foram adicionados 500 ppm de cada um dos padrões de dextranas com Mw 5,90 x 10 $4,86 \times 10^{4}$ e $1,16 \times 10^{4} \mathrm{Da}$. Após $24 \mathrm{~h} \mathrm{a} 4{ }^{\circ} \mathrm{C}$, estas soluções foram filtradas e analisadas. Comparando-se as áreas de cada padrão antes e após a filtração verificou-se que este procedimento eliminou 83,3\% das dextranas de Mw 5,90 x $10^{6}$, e somente de 5,0 a 12,1\% das demais dextranas $\left(\mathrm{Mw}=4,86 \times 10^{4}\right.$, e $\left.1,16 \times 10^{4} \mathrm{Da}\right)$.

Os resultados acima indicam que procedimentos como o resfriamento e a posterior filtração da cachaça, nas condições do ensaio, não são suficientes para retirar as dextranas, principalmente a fração de menor massa molar.

Apesar da maior parte do conteúdo da fração de massa molar mais elevada ser retida por filtração, a concentração remanescente $(16,7 \%)$ ainda poderá desencadear a formação de flocos. Além de possuir menor solubilidade em etanol, estas dextranas por apresentarem um maior número de ramificações possuem uma maior capacidade de se ligar a metais, como o cobre e o ferro. ${ }^{1,10,11}$
As dextranas presentes nas frações de menor massa molar, cuja presença é pouco alterada pela filtração em função da sua concentração no açúcar utilizado, também poderão desencadear a formação de flocos conforme já relatado em soluções parenterais de dextrana com massa molar de 4,00 x $10^{4}$ ou 7,00 x $10^{4} \mathrm{Da}^{26}$

Atualmente nosso laboratório realiza estudos que buscam contribuir para o entendimento da dinâmica da formação de precipitados, em função das diferentes frações de dextrana presentes em amostras de açúcares e do perfil químico da cachaça.

\section{CONCLUSÕES}

As amostras de açúcar analisadas indicam a presença de dextranas de massa molar entre $10^{6}$ e $10^{4} \mathrm{Da}$, e que somente o conhecimento do teor de dextranas totais em açúcares pode não ser suficiente para a prevenção do surgimento de precipitados em soluções hidroalcoólicas. Assim, será de grande utilidade para os produtores de açúcar, de cachaça e indústria alimentícia, conhecerem além do teor de dextranas totais, também o perfil da sua distribuição em termos de massa molar.

\section{AGRADECIMENTOS}

À CAPES, ao CNPq e à FAPESP pelo apoio financeiro e ao Centro de Tecnologia Canavieira (Piracicaba-SP) na pessoa do sr. E. da Silva pelas amostras.

\section{REFERÊNCIAS}

1. Rodrigues, S.; Tese de Doutorado, Universidade Estadual de Campinas, Brasil, 2003

2. Singleton, V.; Int. Sugar J. 2002, 104, 132.

3. Eggleston, G.; Legendre, B.; Tew, T.; Food Chem. 2004, 87, 119.

4. Ravno, A. B.; Purchase, B. S.; Int. Sugar J. 2006, 108, 255.

5. Naessens, M.; Cerdobbel, A.; Soetaert, W.; Vandamme, E. J.; J. Chem. Technol Biotechnol. 2005, 80, 845 .

6. Kim, D.; Robyt, J. F.; So-Young, L.; Jin-Ha, L.; Young-Min, K.; Carbohydr. Res. 2003, 338, 1183.

7. Ioan, C. E.; Aberle, T.; Burchard, W.; Macromolecules 2000, 33, 5730.

8. Singleton, V.; Horn, J.; Bucke, C.; Adlard, M.; Int. Sugar J. 2001, 102, 251 .

9. Clarke, M. A.; Roberts, E. J.; Godshall, M. A.; Carpenter, F. G.; Int. Sugar J. 1978, 80, 197.

10. Hirata, Y.; Aoki, M.; Kobatake, H.; Yamamoto, H.; Biomaterials 1999, 20, 303 .

11. Hirata, Y.; Sano, Y.; Aoki, M.; Kobatake, H.; Kato, S.; Yamamoto, H.; J. Colloid Interface Sci. 1999, 212, 530.

12. Roberts, E. J.; Int. Sugar J. 1983, 85, 10.

13. Brasil; Instrução Normativa $n^{\circ} 13$, de 29 de junho de 2005, Ministério da Agricultura Pecuário e Abastecimento, Diário Oficial da União - seção 1, p.3-4, de 30 de junho de 2005.

14. Jackson, D. S. Em Encyclopedia of Food Sciences and Nutrition; Caballero, B.; Trugo, L.; Finglas, P., eds.; Academic Press: Oxford, 2003, vol. 9, p. 5561.

15. Ratnayake, W. S.; Jackson, D. S.; J. Agric. Food Chem. 2006, 54, 3712.

16. Irvine, J. E. Em Cane Sugar Handbook; Chen, J. C. P., ed.; WileyIntersciene: New York, 1985, cap 1.

17. European Pharmacopoeia, 1997, $3^{\mathrm{a}}$ ed., Identification test B, 1369.

18. Canevarolo Júnior, S. V.; Técnicas de Caracterização de Polímeros, Artliber Editora: São Paulo, 2004, cap. 6.

19. Khalikova, E.; Sussi, P.; Korpela, T.; Microbiol. Mol. Biol. Rev. 2005, 69. 306.

20. Mehvar, R.; J. Controlled Release 2000, 69, 1.

21. Brown, C. F.; Inkerman, P. A.; J. Agric. Food Chem. 1992, 40, 227.

22. Clarke, M.; A.; STAB 2000, 18, 48.

23. Chmelík, J.; Chmelíková, J.; Novotny, M. V.; J. Chromatogr., A 1997, 790, 93.

24. Kuge, T; Kobayashi, K.; Kitamura, S; Tanahashi, H.; Carbohydr Res. 1987, $160,205$.

25. Novaes, F. V.; Comunicação pessoal.

26. Pitz, H.; Anaesthesist 1977, 26, 569. 